\title{
Socioeconomic gradients in cardiovascular risk in Canadian children and adolescents
}

\author{
Y. Shi, MD, PhD; M. de Groh, PhD; C. Bancej, PhD
}

This article has been peer reviewed.

Tweet this article

\begin{abstract}
Introduction: Cardiovascular disease (CVD) and its risk factors show clear socioeconomic gradients in Canadian adults. Whether socioeconomic gradients in cardiovascular risk emerge in childhood remains unclear. The objective of this study was to determine whether there are socioeconomic gradients in physiological markers of CVD risk in Canadian children and adolescents.
\end{abstract}

Methods: Using combined cross-sectional data from the Canadian Health Measures Survey 2007-2011, we examined the following cardiovascular risk markers: overweight (including obesity), aerobic fitness score (AFS), blood pressure (BP), blood lipids (total as well as HDL and LDL cholesterol and triglycerides), glucose metabolism and C-reactive protein (CRP) by sex in 2149 children (ages 6-11 years) and 2073 adolescents (ages 12-17 years). Multivariate linear and logistic regression analyses were used to identify patterns in cardiovascular risk across strata of household income adequacy and parental educational attainment, adjusting for age and ethnicity, and stratified by age group and sex.

Results: Young boys showed markedly higher prevalence of obesity than young girls (prevalence of $18.5 \%$, $95 \%$ confidence interval [CI]: 15.6-21.5 vs. 7.7\%, 95\% CI: 5.2-10.3). However, negative SES gradients in adiposity risk were seen in young and adolescent girls rather than boys. Young and adolescent boys were more physically fit than girls (mean AFS of 541, $95 \%$ CI: $534-546$ vs. $501,95 \%$ CI: $498-505$ in children; $522,95 \%$ CI: $514-529$ vs. $460,95 \%$ CI: $454-466$ in adolescents; $p<.001$ ). Although a positive income gradient in AFS was observed in both boys and girls, statistical significance was reached only in girls $(p=.006)$. A negative gradient of parental education in BP was observed in young children. While we observed substantial sex differences in systolic BP, total and HDL cholesterol, fasting glucose and CRP in adolescents, sex-specific socioeconomic gradients were only observed for systolic BP, HDL and LDL cholesterol. Further studies with large samples are needed to confirm these findings.

Conclusion : This study identified important sex difference and socioeconomic gradients in adiposity, aerobic fitness and physiological markers of CVD risk in Canadian schoolaged children. Population health interventions to reduce socioeconomic gradients in CVD risk should start in childhood, with a particular focus on preventing obesity in young boys of all SES and girls of low SES, promoting physical fitness especially in girls and in all ages of youth in low-SES groups, and increasing parental awareness, especially those with low educational attainment, of early CVD risks in their children.

Keywords: socioeconomic gradients, socioeconomic status, cardiovascular risk, physical fitness, obesity, children and adolescents, Canadian Health Measures Survey
Key findings

- Young boys had higher prevalence of obesity than young girls.

- Boys were more physically fit than girls.

- Canadian children and adolescents, particularly girls, show significant socioeconomic gradients in obesity, physical fitness and several physiological markers of risk of cardiovascular disease.

\section{Introduction}

Cardiovascular disease (CVD) is the leading cause of death in Canadian adults. ${ }^{1}$ In adults, CVD and its risk factors show clear socioeconomic gradients. $^{2,3}$ Physiological and behavioural risk factors associated with CVD include overweight (as well as obesity), elevated blood pressure (BP), elevated lowdensity lipoprotein (LDL) and low highdensity lipoprotein (HDL), elevated blood glucose, smoking, physical inactivity/low physical fitness and consuming a high fat diet. ${ }^{4}$ Behavioural factors are known to be associated with many adverse health outcomes including the development of physiological risk factors of CVD. ${ }^{5}$ Socioeconomic status (SES) is used as a proxy of physical environments in which children live and play. Emerging evidence suggests that several adverse health behaviours associated with CVD risk, such as unhealthy eating, physical inactivity and smoking, are disproportionally higher in youth with low SES. ${ }^{6-8}$ Other research suggests children's long-term health can be affected by biological embedding

Author reference:

Social Determinants and Science Integration Directorate, Public Health Agency of Canada

Correspondence: Yipu Shi, Social Determinants and Science Integration Directorate, Public Health Agency of Canada, Ottawa, ON K1A 0K9; Tel: 613-941-2436; Fax: 613-960-0921;

Email: yipu.shi@phac-aspc.gc.ca 
of adversity during sensitive developmental periods and that children with low SES may be especially vulnerable to stressful influences. ${ }^{9}$ We need further evidence to understand whether socioeconomic gradients in physical health develop in children exposed to various socioeconomic circumstances.

There is ample evidence that CVD risk factors originate in childhood and that low SES in childhood is associated with elevated risk of CVD and increased CVD mortality in later life. ${ }^{10,11}$ SES is frequently measured as family income, parental education and occupational status. Family income and parental education, each representing a separate dimension of SES, are among the social factors most strongly associated with health. ${ }^{12}$ Income may influence health most directly through access to material resources; education may be directly related to health through health behaviours and lifestyle choices and indirectly through income and psychosocial factors. ${ }^{13}$ The direct and indirect contribution of material factors strongly predicts health in children. Intergenerational studies have found that parents' education can have a substantial impact on the health and education outcomes of their children. ${ }^{14}$ Assessing the independent effect of income and education on health may help us understand the mechanisms through which they influence health and provide more options for policy development to reduce the risk of poor health outcomes for children.

Given the childhood obesity epidemics in many countries, a number of studies have demonstrated socioeconomic gradients in relation to obesity and, as a result, interventions aimed at reducing inequalities in childhood obesity have been created and implemented. ${ }^{15,16}$ While recent evidence suggests a sex-specific pattern of SES gradients in overweight in Canadian adults, studies in children have not identified any such differences in SES-related overweight risk. ${ }^{8,17}$ Few studies have addressed the early emergence of SES gradients in physiological CVD markers, and findings have been inconsistent partly due to differences in the SES indicators and in the age ranges of the study populations. $^{18-20}$ Understanding socioeconomic impacts on CVD risks in childhood may help identify high-risk groups to target for early CVD prevention programs that prevent lifelong inequalities in CVD.
The Canadian Health Measures Survey (CHMS), ${ }^{21}$ a nationally representative survey with physical measures and blood and urine collection, provides a unique opportunity to examine the association of SES with physiological markers of CVD risk in Canadian children and adolescents.

\section{Methods}

\section{Data source}

The CHMS is a cross-sectional, comprehensive health measures survey that collects information on health status and risk factors in the Canadian household population. The survey represented $96.3 \%$ of the Canadian population aged 6 to 79 years living at home in the 10 provinces and 3 territories; it excludes people living on reserves or other Aboriginal settlements, certain remote areas and institutions, and full-time members of the Canadian Armed Forces. The Health Canada Research Ethics Board reviewed and approved all CHMS processes and protocols. Participation in the survey was voluntary and informed consent was obtained from each participant. We combined Cycle 1 (20072009) and Cycle 2 (2009-2011) for this study; the overall response rate for both cycles combined was $53.5 \%{ }^{21}$

The surveys consisted of a household interview that includes questions on sociodemographic characteristics and health and lifestyle and a visit to a mobile examination centre where physical measures are made and blood and urine samples collected. Parents/guardians answered all questions for participants aged 6 to 13 years; participants aged 14 years and older answered all questions on their own. Approximately half of the respondents were randomly selected to fast overnight before blood samples were taken. To increase statistical power, we combined CHMS Cycles 1 and 2 for a sample of 3799 (and a fasting sample of 1693) respondents aged 6 to 17 with data collected between 2007 and 2011

\section{Measures}

\section{Socioeconomic status}

We used household income adequacy and parental educational attainments as indicators of SES. Household income adequacy is a variable derived by Statistics Canada, calculated using both total family income from all sources and total number of household members, and classified into lowest, lower-middle, upper-middle and highest income groups. Because only about $70 \%$ of the CHMS respondents reported their total household income, Statistics Canada used regression modelling techniques to impute missing values based on all or part of the following information: partial responses for the income range, nearest neighbour, collection site and household size. $^{22}$ We chose to only include respondents whose imputed income was based on fully or partially reported income range.

Parental educational attainment was based on the highest education attained by either parent, and categorized as less than secondary, secondary, some post-secondary and completed post-secondary education. Ethnicity was defined as White or non-White, the latter included Aboriginals living off-reserve. Respondents with missing values on these variables were excluded from the analysis, resulting in a total sample of 3591 and a fasting sample of 1645 .

\section{Cardiovascular outcomes}

Birth weight of the CHMS participants aged 6 to 11 years was reported by their guardians. These children's physical activity was determined from their guardians' answers to the question "Over a typical or usual week, on how many days was he/she physically active for a total of at least 60 minutes per day?" Available answers included: (1) none; (2) 1 day; (3) 2 to 3 days; (4) 4 or more days. The child was considered physically active if the answer was (4); otherwise, they were considered inactive. ${ }^{22}$ For adolescents aged 12 to 17, the physical activity module for adults was adopted and levels of activity classified as active, moderately active and inactive. $^{22,23}$ Cigarette smoking in adolescents was defined as current (combined current daily or occasional smoker) versus non-smoker (never smoked).

Body mass index (BMI) was calculated using measured standing height and weight, and weight status was defined according to World Health Organization growth reference for school-aged children and adolescents. ${ }^{24}$ Waist circumference was measured at the mid-point between the highest point of the iliac crest and the last floating rib. ${ }^{22}$ 
Aerobic fitness or cardiorespiratory fitness levels were determined using the modified Canadian Aerobic Fitness Test (mCAFT) by recording participants' age-predicted maximal heart rate and calculating their predicted maximal aerobic power $\left(\mathrm{VO}_{2} \max \right){ }^{25}$ The definition of poor aerobic fitness was based on a derived variable indicating aerobic fitness within a range that is generally associated with certain health risks in children. Details of the derivation of aerobic fitness score (AFS) and aerobic fitness norm are published in methodological papers and the CHMS Data Users' Guide. ${ }^{22,26,27}$ Resting systolic BP and diastolic BP were measured according to the new protocol for standard BP measurement in surveys, described in our previous publication. ${ }^{28}$

All laboratory assays were conducted by Health Canada. Details of the standard laboratory procedures are available online. ${ }^{22}$ Values for laboratory variables that were below the limit of detection were replaced by limit of detection divided by two. The dependent variables for CVD markers in the full sample included total cholesterol (mmol/L), HDL (mmol/L) and C-reactive protein (CRP; mg/L), while those based on the fasting sub-sample included LDL (mmol/L), triglycerides (mmol/L), glucose (mmol/L) and insulin (pmol/L). Insulin resistance was estimated by homeostasis model assessment of insulin resistance (HOMA-IR), a surrogate measure of insulin resistance in non-diabetic children, calculated by dividing the product of $0.1394 *$ insulin ( $\mathrm{pmol} / \mathrm{ml}$ ) and glucose $(\mathrm{mmol} / \mathrm{ml})$ by $22.5 .^{29}$ To preserve the size of the sample, respondents with missing values for a given dependent variable were excluded only from analyses involving that variable.

\section{Statistical analyses}

Respondents' SES and CVD risk characteristics were described for children and adolescents and compared between boys and girls using t-tests. We first examined the polychoric correlation between household income adequacy and parental educational attainment. Since they were only moderately correlated (correlation coefficient $=0.45$ ), the independent effect of income adequacy and parental education on CVD risk were examined by (1) multivariate logistic regression for overweight (including obesity) and poor aerobic fitness, and (2) multivariate linear regression for BMI, waist circumference, AFS, BP, blood lipids (total, HDL and LDL cholesterol and triglycerides), fasting glucose, insulin and HOMA-IR, and CRP, adjusting for age and ethnicity, and stratified by sex. For the associations of SES with BP, models additionally adjusted for heart rate and height. Depending on the age range, we did not control for birth weight and physical activity in children, or smoking status and physical activity in adolescents because these variables may act as mediators so that the effects of SES on CVD risk may be underestimated.

Because of the complex sampling design of the CHMS and limited number of primary sampling units of Cycles 1 and 2, bootstrap weights were applied for variance estimation for proportions, means and parameters of regression models, with 24 degrees of freedom specified for combined Cycle 1 and 2 data. We used multivariate logistic and linear regression models to examine trends in CVD risks across all strata of income adequacy and parental education, adjusted for age and ethnicity. Satterthwaite-adjusted chi-square statistics were used to determine statistical significance $(p<.05)$ of a linear trend.

All analyses were performed using SAS version 9.3 (SAS Institute Inc., Cary, NC, USA) and SUDANN version 10.0.1 (RTI International, Research Triangle Park, NC, USA).

\section{Results}

Table 1 shows SES indicators and CVD risk factors by sex in children aged 6 to 11 years. Boys were born significantly heavier than were girls. Prevalence of obesity in boys $(18.5 \%$; $95 \%$ confidence interval [CI]: 15.6-21.5) was more than double that of girls $(7.7 \%$; $95 \%$ CI: $5.2-10.3$; $p<.001)$. Similarly, average waist circumference was greater in boys than in girls (62.5 cm; $95 \%$ CI: $61.7-63.0$ vs. $60.4 \mathrm{~cm}$; 95\% CI: 59.7-61.5; $p=.0004)$.

Boys were physically fitter than girls, with a higher mean AFS (541; $95 \%$ CI: 534-546 vs. 501 ; $95 \%$ CI: $498-505$; $p<.001)$ and a lower prevalence of poor aerobic fitness
(23.2; $95 \%$ CI: $18.5-28.7$ vs. 30.6 ; $95 \%$ CI: $26.3-35.3 ; p=.04)$. No sex differences were found in most CVD physiological markers except for mean fasting insulin, which was higher in girls than boys (54.6 pmol/L; 95\% CI: 50.0-59.2 vs. 43.4 pmol/L; $95 \%$ CI: 37.6-49.2; $p=.03$ ). We found no marked sex differences in the SES indicators.

Table 2 shows SES indicators and CVD risk factors by sex in adolescents aged 12 to 17 years. The sex difference in aerobic physical fitness persisted and increased in the adolescent population, with adolescent girls having even lower AFS (460; $95 \%$ CI: 454-466 vs. 522 ; $95 \%$ CI: $514-529$ for adolescent boys; $p<.001$ ) and a higher proportion of poor aerobic fitness than adolescent boys (18.0; 95\% CI: 14.7-21.9 for girls vs. 8.60; $95 \%$ CI: 5.97-12.3; $p=.002$ ). Moreover, we observed marked sex differences in multiple CVD markers such as unfavorable systolic BP, fasting glucose and HDL in adolescent boys, and unfavorable total cholesterol and CRP levels in adolescent girls. Again, no marked sex differences in SES indicators were observed.

Table 3 shows gradients of CVD risk in children according to income adequacy and parental educational attainment. For sufficient statistical power, we combined overweight and obese groups as a dependent variable in regression analyses. We found a significant income gradient in BMI ( $p$ for trend:.006) and overweight ( $p$ for trend:.01) in young girls, whereas nonWhite young boys showed a higher overweight risk than White boys (OR: 1.55; 95\% CI: 1.03-2.32). Moreover, we also observed income and educational gradients in aerobic fitness (mean AFS and proportion of poor aerobic fitness) in young girls ( $p$ for trend:.006 and.003, respectively); a similar trend of income gradient in aerobic fitness was seen in young boys but did not reach statistical significance ( $p$ for trend:.11). Regardless of sex, there were negative educational gradients in elevated systolic BP and diastolic BP in young children. Non-White children had higher mean HDL than White children ( $\beta=0.11$; 95\% CI: 0.04-0.18). Moreover, we also observed a positive parental educational gradient in HDL in young girls ( $p$ for trend:.047). 
TABLE 1

Socioeconomic status and cardiovascular risk of Canadian children, 6-11 years, by sex

\begin{tabular}{|c|c|c|c|c|c|}
\hline & \multirow[t]{2}{*}{ Sample size, $\mathbf{n}^{\mathbf{b}}$} & \multicolumn{3}{|c|}{ Percentage or mean $(95 \% \mathrm{Cl})^{\mathrm{a}}$} & \multirow[t]{2}{*}{$p$ value } \\
\hline & & Total & Boys & Girls & \\
\hline Income adequacy & 2073 & & & & \\
\hline Lowest & 128 & $5.96(4.31-8.19)$ & $5.62(3.74-8.37)$ & $6.34(4.21-9.44)$ & .62 \\
\hline Lower-middle & 349 & $19.4(16.1-23.2)$ & $18.7(14.1-24.4)$ & $20.2(16.4-24.5)$ & .62 \\
\hline Upper-middle & 569 & $27.6(24.6-30.8)$ & $26.5(23.4-29.8)$ & $28.8(24.0-34.1)$ & .40 \\
\hline Highest & 1027 & $47.1(41.9-52.3)$ & $49.2(42.8-55.6)$ & $44.7(39.4-50.1)$ & .12 \\
\hline Parental education & 2073 & & & & \\
\hline Less than secondary & 80 & $3.89(2.62-5.72)$ & $3.55(2.36-5.31)$ & $4.27(2.43-7.38)$ & .54 \\
\hline Secondary & 178 & $8.75(6.79-11.2)$ & $8.02(5.18-12.2)$ & 9.59 (7.34-12.4) & .45 \\
\hline Some post-secondary & 93 & $4.29(2.84-6.45)$ & $4.90(2.64-8.92)$ & $3.61(2.16-5.97)$ & .46 \\
\hline Post-secondary & 1722 & $83.1(79.6-86.0)$ & $83.5(78.3-87.7)$ & $82.5(78.7-85.7)$ & .71 \\
\hline Ethnicity - White & 1575 & $72.7(62.5-81.0)$ & $74.2(63.7-82.5)$ & $71.1(60.2-79.9)$ & .23 \\
\hline Birth weight, g & 2020 & 3378 (3338-3418) & 3441 (3379-3503) & 3312 (3264-3360) & .002 \\
\hline Physically active & 1735 & $83.1(81.0-85.2)$ & $83.2(79.9-86.5)$ & $83.1(80.6-85.5)$ & .71 \\
\hline \multicolumn{6}{|l|}{ Cardiovascular risk } \\
\hline $\mathrm{BMI}, \mathrm{kg} / \mathrm{m}^{2}$ & 2058 & 17.9 (17.7-18.1) & $18.2(17.9-18.4)$ & $17.6(17.4-17.9)$ & .003 \\
\hline Overweight & 2058 & $20.4(17.4-23.7)$ & $18.8(15.2-23.1)$ & $22.1(18.7-26.0)$ & .11 \\
\hline Obese & 2058 & $13.4(11.3-15.7)$ & $18.5(15.6-21.5)$ & $7.7(5.2-10.3)$ & $<.001$ \\
\hline Waist circumference, cm & 2058 & $61.5(61.0-62.2)$ & $62.5(61.7-63.0)$ & $60.4(59.7-61.5)$ & $<.001$ \\
\hline AFS & 1302 & $518(516-521)$ & $541(534-546)$ & $501(498-505)$ & $<.001$ \\
\hline Poor aerobic fitness & 1302 & $27.0(23.9-30.3)$ & $23.2(18.5-28.7)$ & $30.6(26.3-35.3)$ & .04 \\
\hline Systolic BP, mmHg & 2063 & 93.9 (93.4-94.3) & $93.6(93.0-94.3)$ & 94.2 (93.6-94.8) & .198 \\
\hline Diastolic BP, mmHg & 2063 & $61.0(60.5-61.6)$ & $60.8(60.0-61.6)$ & $61.3(60.7-61.8)$ & .29 \\
\hline C-reactive protein, $\mathrm{mg} / \mathrm{L}$ & 1791 & $0.96(0.80-1.12)$ & $1.05(0.76-1.35)$ & $0.86(0.76-0.96)$ & .2 \\
\hline Total cholesterol, mmol/L & 1816 & $4.23(4.16-4.30)$ & $4.22(4.13-4.31)$ & $4.25(4.16-4.33)$ & .66 \\
\hline HDL cholesterol, mmol/L & 1816 & $1.42(1.39-1.45)$ & $1.44(1.40-1.47)$ & $1.40(1.36-1.44)$ & .11 \\
\hline LDL cholesterol, mmol/L & 887 & $2.35(2.28-2.43)$ & $2.36(2.26-2.46)$ & $2.35(2.22-2.47)$ & .88 \\
\hline Log-triglyceride, mmol/L & 887 & $0.82(0.77-0.87)$ & $0.79(0.73-0.85)$ & $0.85(0.79-0.92)$ & .12 \\
\hline Glucose, mmol/L & 879 & $4.60(4.51-4.69)$ & $4.67(4.53-4.81)$ & $4.53(4.46-4.60)$ & .05 \\
\hline Insulin, pmol/L & 856 & $48.9(45.2-52.6)$ & $43.4(37.6-49.2)$ & $54.6(50.0-59.2)$ & .03 \\
\hline HOMA-IR & 851 & $1.56(1.31-1.82)$ & $1.46(1.02-1.91)$ & 1.67 (1.41-1.93) & .6 \\
\hline
\end{tabular}

Source: 2007-2011 Canadian Health Measures Survey.

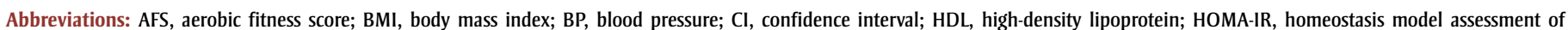
insulin resistance; LDL, low-density lipoprotein.

a Population-weighted percentage or mean; 95\% confidence interval.

${ }^{\mathrm{b}}$ Depending on the variable, analysis excludes 76 to 333 non-respondents from the full sample and 26 to 62 non-respondents from the fasting sample.

Table 4 shows gradients in CVD risk in adolescents according to income adequacy and parental education. In contrast to the lack of SES gradient in risk of overweight in young boys, we observed a positive income gradient in adolescent boys, with boys living in the highest income households showing the highest risk. We observed a similar gradient in income adequacy when BMI was analyzed as a continuous outcome variable. There was an educational gradient in overweight risk in adolescent girls, with overweight prevalence higher in girls whose parents had low educational attainment. SES gradients in aerobic fitness persisted in girls into adolescence ( $p$ for trend:.05).

In terms of other physiological markers of CVD risk, we observed gradients of income adequacy in systolic BP and in total and LDL cholesterol in adolescent boys, with boys in highest income households demonstrating the highest risk, which is the same direction of income gradient observed with overweight. Furthermore, there were educational gradients in LDL cholesterol in adolescent boys and income gradient in HDL cholesterol in adolescent girls, with low-SES adolescents showing the higher risk. Similar to what we observed in younger children, non-White adolescents had higher mean HDL cholesterol than did their White counterparts ( $\beta=0.08,95 \% \mathrm{CI}$ : $0.01-0.16)$. Even though we found significant trends in some of the CVD markers estimated 
TABLE 2

Socioeconomic status and cardiovascular risks of Canadian adolescents, 12-17 years, by sex

\begin{tabular}{|c|c|c|c|c|c|}
\hline & \multirow[t]{2}{*}{ Sample size, $\mathbf{n}^{\mathbf{b}}$} & \multicolumn{3}{|c|}{ Percentage or mean $(95 \% \mathrm{CI})^{\mathrm{a}}$} & \multirow[t]{2}{*}{$p$ value } \\
\hline & & Total & Boys & Girls & \\
\hline Income adequacy & 1518 & & & & \\
\hline Lowest & 73 & $5.05(3.56-7.12)$ & $4.22(2.63-6.70)$ & 5.99 (3.81-9.31) & .25 \\
\hline Lower-middle & 215 & $14.7(11.9-18.1)$ & $17.0(13.3-21.5)$ & $12.1(8.6-16.8)$ & .07 \\
\hline Upper-middle & 443 & $27.7(23.3-32.7)$ & $27.6(21.6-34.7)$ & $27.8(22.4-34.0)$ & .96 \\
\hline Highest & 787 & $52.5(46.3-58.6)$ & $51.1(43.8-58.4)$ & $54.0(46.5-61.3)$ & .47 \\
\hline Parental education & 1518 & & & & \\
\hline Less than secondary & 52 & $3.83(2.08-6.94)$ & $3.03(1.15-7.78)$ & $4.74(2.68-8.24)$ & .28 \\
\hline Secondary & 129 & $9.38(7.11-12.3)$ & $10.2(7.46-13.8)$ & $8.45(5.42-12.9)$ & .42 \\
\hline Some post-secondary & 117 & $7.54(5.58-10.1)$ & $7.26(4.75-11.0)$ & $7.86(5.58-10.9)$ & .74 \\
\hline Post-secondary & 1220 & $79.2(75.0-82.9)$ & 79.5 (73.7-84.2) & $79.0(73.4-83.6)$ & .87 \\
\hline Ethnicity - White & 1620 & $74.6(65.4-82.0)$ & $74.1(64.6-81.9)$ & $75.1(64.7-83.3)$ & .76 \\
\hline Physically active & 1505 & $30.1(26.8-33.7)$ & $31.5(27.4-35.9)$ & $28.6(23.1-34.7)$ & .42 \\
\hline Daily smoker & 1518 & $4.63(2.98-7.12)$ & $5.62(2.95-10.5)$ & $3.50(2.23-5.45)$ & .28 \\
\hline \multicolumn{6}{|l|}{ Cardiovascular risk } \\
\hline $\mathrm{BMI}, \mathrm{kg} / \mathrm{m}^{2}$ & 1518 & $21.9(21.4-22.5)$ & $21.9(21.2-22.6)$ & $22.0(21.3-22.7)$ & .86 \\
\hline Overweight & 1518 & $18.3(15.1-22.0)$ & 17.9 (13.9-22.7) & $18.8(14.6-23.8)$ & .8 \\
\hline Obese & 1518 & $14.3(11.2-18.2)$ & $13.9(10.1-18.7)$ & $14.8(10.4-20.8)$ & .2 \\
\hline Waist circumference, cm & 1518 & $75.2(73.9-76.4)$ & 75.9 (74.2-77.7) & $74.2(72.4-75.9)$ & .13 \\
\hline AFS & 1518 & $492(487-497)$ & $522(514-529)$ & $460(454-466)$ & $<.001$ \\
\hline Poor aerobic fitness & 1518 & $13.1(11.3-15.1)$ & $8.60(5.97-12.3)$ & $18.0(14.7-21.9)$ & .002 \\
\hline Systolic BP, mmHg & 1514 & 98.0 (97.1-98.9) & $99.7(98.4-101.0)$ & $96.1(95.3-96.8)$ & $<.001$ \\
\hline Diastolic BP, mmHg & 1514 & $61.8(60.9-62.8)$ & $62.4(60.7-63.3)$ & $61.6(60.8-62.4)$ & .45 \\
\hline C-reactive protein, $\mathrm{mg} / \mathrm{L}$ & 1389 & $0.97(0.83-1.12)$ & $0.82(0.70-0.94)$ & $1.15(0.87-1.42)$ & .036 \\
\hline Total cholesterol, mmol/L & 1423 & $4.06(3.98-4.13)$ & $3.99(3.91-4.08)$ & $4.14(4.04-4.23)$ & .005 \\
\hline HDL cholesterol, mmol/L & 1423 & $1.31(1.28-1.34)$ & $1.26(1.23-1.29)$ & $1.37(1.34-1.41)$ & $<.001$ \\
\hline LDL cholesterol, mmol/L & 892 & $2.30(2.21-2.39)$ & $2.31(2.20-2.43)$ & $2.29(2.18-2.40)$ & .72 \\
\hline Log-triglyceride, mmol/L & 892 & $0.96(0.91-1.02)$ & $0.95(0.88-1.03)$ & $0.97(0.90-1.04)$ & .69 \\
\hline Fasting Glucose, mmol/L & 890 & $4.69(4.61-4.77)$ & 4.78 (4.69-4.87) & $4.60(4.52-4.68)$ & $<.001$ \\
\hline Insulin, pmol/L & 869 & $70.2(65.6-74.6)$ & $69.5(62.1-76.9)$ & $70.8(66.4-75.3)$ & .74 \\
\hline HOMA-IR & 867 & $2.07(1.91-2.23)$ & $2.10(1.87-2.32)$ & $2.05(1.89-2.21)$ & .65 \\
\hline
\end{tabular}

Source: 2007-2011 Canadian Health Measures Survey.

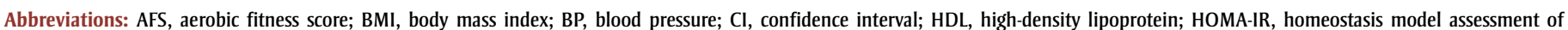
insulin resistance; LDL, low-density lipoprotein.

a Population-weighted percentage or mean; $95 \% \mathrm{CI}$.

b Depending on the variable, analysis excludes 132-227 non-respondents from the full sample and 22-45 non-respondents from the fasting sample.

using fasted samples, these results should be interpreted with caution because of the low sample sizes in low-SES groups.

\section{Discussion}

Our study examined whether there were SES gradients in physiological markers of CVD risk in a nationally representative sample of Canadian children and adolescents. We identified important sex and SES gradients in adiposity and aerobic fitness that emerge early in childhood. Young boys were twice as likely to be obese than young girls; however, decreasing risk of overweight with socioeconomic affluence was only seen in girls. More importantly, we found SES gradients in aerobic fitness throughout childhood, especially in girls. Educational gradients in BP emerged early in childhood. While adolescent boys in affluent families showed higher risk in some physiological markers of CVD, we found SES gradients in decreased HDL in adolescent girls and increased LDL in adolescent boys, with low-SES adolescents showing the higher risk.

Many of the studies that examined the relationship between SES and overweight in children and adolescents from developed countries have found an inverse gradient between SES and overweight. ${ }^{30}$ Our finding on the inverse association of income adequacy with risk of overweight is congruent 


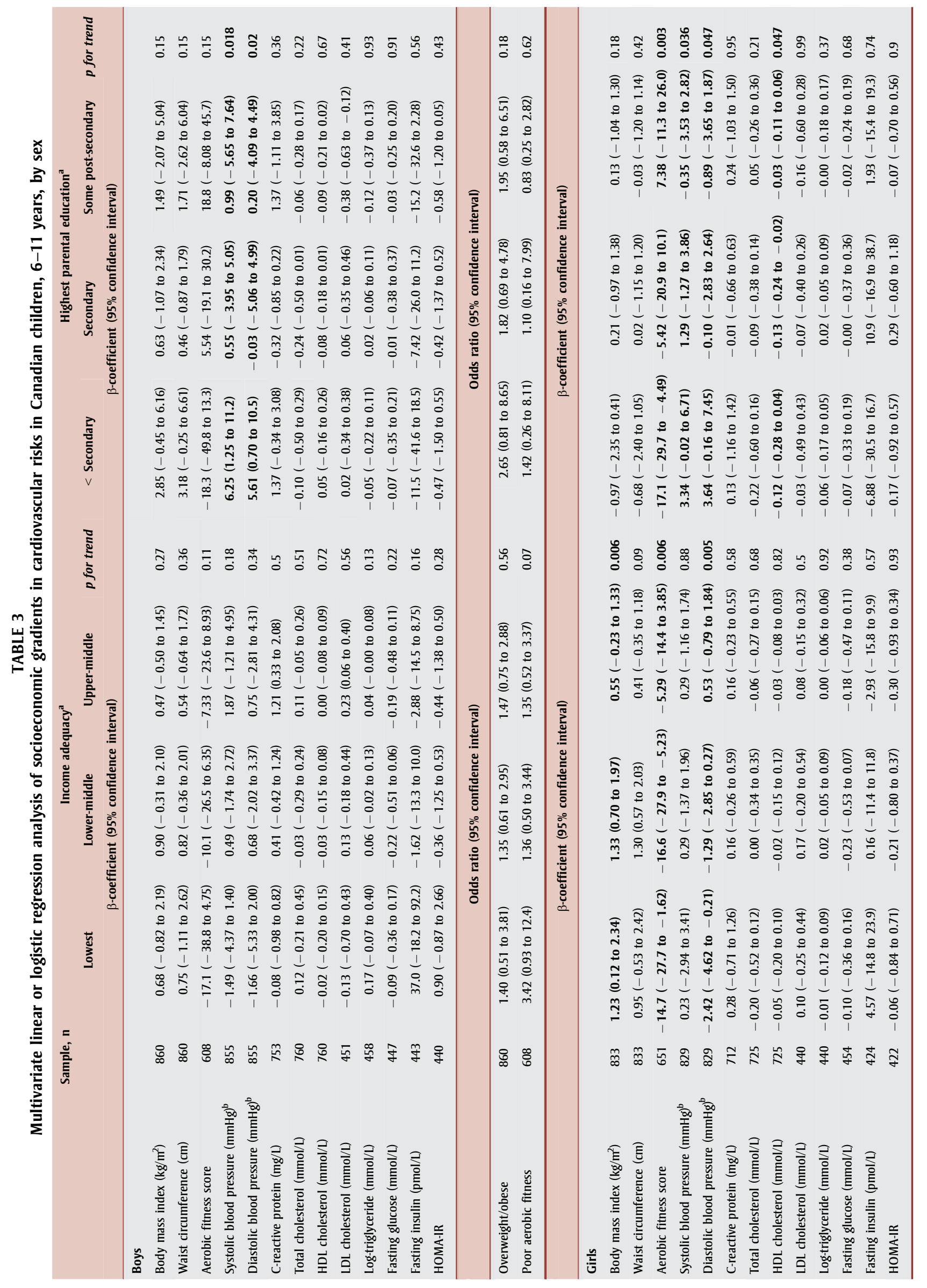




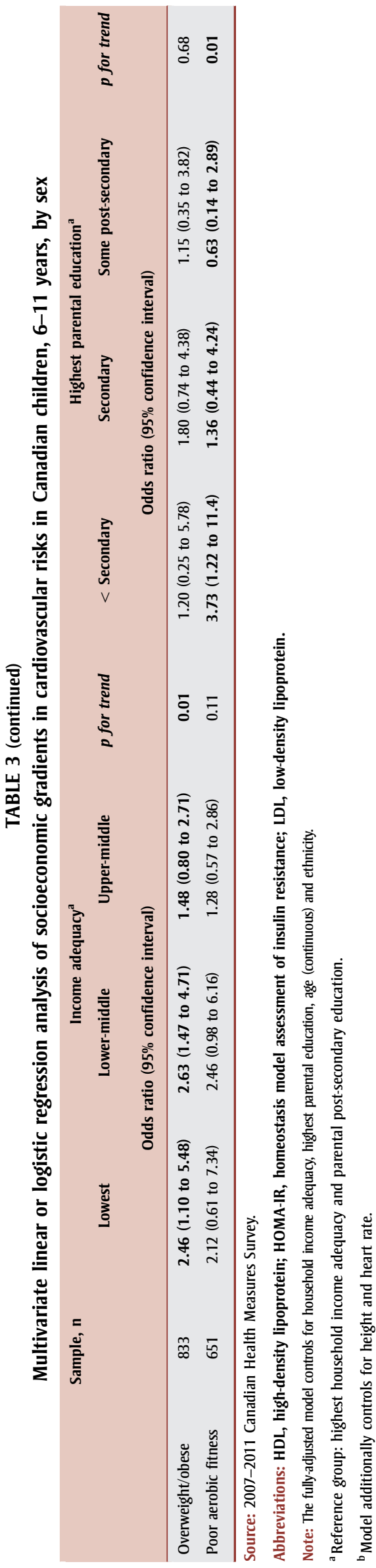

with other Canadian studies. A study of children in Grades 6 to 10 found that both individual- and area-level SES measures were associated with obesity, ${ }^{7}$ and a study of Grade 5 students in a Nova Scotia school reported similar findings, ${ }^{16}$ though Shields et al. ${ }^{31}$ did not find such an association when using national survey data.

None of these studies of SES and overweight in children conducted sex-specific analyses. Our results-a sex-specific pattern, with gradients of income adequacy more strongly associated with overweight in young girls than in boys-mirror findings in recent Canadian studies of adults in which SES gradients in overweight risk were stronger in women than in men. ${ }^{32,33}$ That children from families living in low income have limited access to material resources and are less able to afford leisure activities like organized sports is well understood; also accepted is that participating in organized sports promotes weight loss. In this study, we could not determine whether girls are more vulnerable to living in a disadvantaged socioeconomic environment or whether such an impact begins early in childhood or, indeed, if girls in high SES groups are more influenced and pressured by social norms to stay slim at an early age. ${ }^{34}$ Furthermore, lack of SES gradients in overweight in young boys should not undermine the high prevalence of obesity in this population. The lack of SES gradient in overweight in young boys may be due to cultural/social attitudes that accept heavier weighted boys across all income groups in some ethnic/racial groups. Further evidence is shown by our finding that boys of nonWhite ethnicity were at higher risk of overweight independent of SES. Our finding supports federal/provincial/territorial governments' focus on initiatives to reduce childhood obesity and maintain healthy weight in children, ${ }^{35}$ and further suggests that interventions to prevent childhood obesity and to reduce SES gradients in obesity should not only target low-SES girls but also boys of all SES groups.

Another key finding of this study is the striking sex differences and SES gradients in aerobic fitness in Canadian children and adolescents. We found a persistent SES gradient in aerobic fitness in Canadian girls throughout their childhood. Aerobic fitness, or the measured AFS we used in this study, is generally considered a physiological outcome of frequent physical activity and an objective marker of this behaviour. ${ }^{20}$ The mechanisms by which low-SES may potentially affect the physical fitness of a child include, but are not limited to, gaps in health education, low-quality nutritive food and poor access to recreational facilities.

To the best of our knowledge, this is the first study to analyze the relationship between SES and physical fitness in Canadian youth. Our findings are similar to studies of $\mathrm{US}^{34}$ and Swedish ${ }^{36}$ youth that reported stronger SES gradients in physical fitness in girls than in boys. There is some evidence that boys are more likely to engage in vigorous leisure-time activity than girls, regardless of their SES, and that girls are more likely to engage in physical activity more in the form of organized sports, for which participation may be more encouraged and supported in high SES groups. ${ }^{37,38}$ This hypothesis is supported by Canadian and other studies that showed that girls in low-SES neighbourhoods engaged in significantly more screen time than did girls who lived in high SES neighbourhoods, a relationship not observed in boys. ${ }^{39,40}$ Note that some studies have suggested that organized sport is one of the best ways to encourage vigorous activity in adolescent girls, ${ }^{41,42}$ and that vigorous physical activity is the best way to achieve cardiorespiratory fitness. Intervention research on effectively promoting physical fitness to reduce SES gradients in Canadian youth, and especially those in low-SES groups, is needed.

Our study identified SES gradients in biomarkers of CVD risk in Canadian children, that is, SES gradients in BP in young children and in HDL and LDL cholesterol levels in adolescents, suggesting the lifelong SES gradients in CVD risks are physiologically identifiable in childhood. Intergenerational research suggests that parents' education may affect children's health indirectly through income or by affecting a child's psychological well-being through poor parenting style or through chronic stress, which may be disproportionally high in children in socioeconomically disadvantaged families. ${ }^{12}$ Our finding of an educational gradient in BP is consistent with the results of other studies that found that low parental education and 


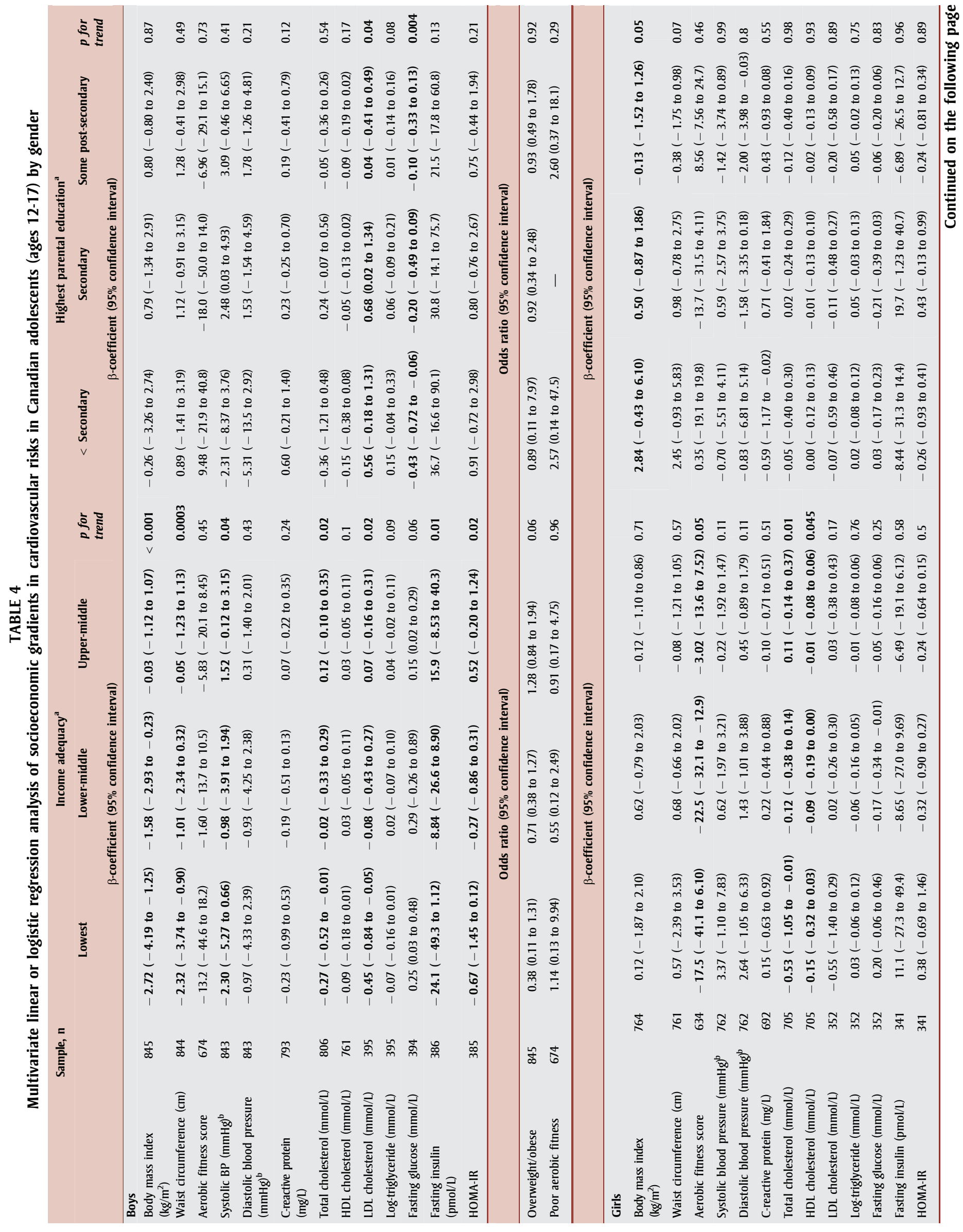


harsh family environments explain some BP variability in children. ${ }^{43,44}$

Studies of the relationship between SES and lipids in children in other populations have produced inconsistent results. ${ }^{45}$ Current evidence suggests that race/ethnicity, physical activity and body weight are important predictors for HDL and foods high in saturated fat, physical activity and body weight are among the determinants for LDL in children and adolescents. ${ }^{46}$ Our finding of a relatively favorable HDL profile in non-Whites independent of SES suggests that some ethnic groups are more genetically protected than others from CVD. Both physical activity and body weight are among the determinants of cholesterol levels at an early age, reinforcing existing public health messages aimed at promoting physical activity and healthy diets and preventing overweight, particularly in socioeconomically disadvantaged children (and adolescents in particular) in order to prevent disparity in cardiovascular risk that could originate in childhood.

\section{Strengths and limitations}

Strengths of this study included our use of a nationally representative sample that provides reliable information on objective measures of CVD risks that allowed us to analyze early effects of SES on physiological markers of CVD risks. A population-based sampling strategy allows the results to be generalized to the Canadian population.

Limitations of our study are the small sample size, especially for low-SES groups and fasting samples, which may have prevented us from detecting an SES gradient or interpreting trends in subpopulations with large measurement variability, and the inability to examine potential interactions between SES indicators. Another limitation was biases associated with self-reported information on family income and parental education. Due to the cross-sectional design of the study, we were unable to assess changes (increases or decreases) of SES gradients in CVD risks over time throughout childhood. Some evidence suggests that childhood SES gradients in health track through adulthood, ${ }^{47}$ whereas others found that SES gradients established in childhood do not persist through adolescence, ${ }^{18}$ these different conclusions suggest the need for further research.

\section{Conclusion}

We sought to determine whether the clear SES gradient in CVD risk observed in Canadian adults is also seen in children and adolescents. We analyzed independent associations of income adequacy and parental education with physiological markers for CVD risks. Our study identified striking sex and SES gradients in adiposity and aerobic fitness in Canadian children, in particular in girls aged 6 to 17 years. Although an SES gradient in adiposity was not apparent in boys, and in fact, an inverse SES gradient in overweight was found in adolescent boys, young boys presented a considerably higher prevalence of obesity and abdominal obesity than do girls. This population should not be overlooked in the efforts to reduce CVD risk in childhood.

The educational gradients in some of the physiological markers that we observed in Canadian youth require further examination to confirm. However, health promotion should consider increasing awareness of early CVD risks in parents, especially less educated parents.

Our findings support current priorities to reduce childhood obesity and health inequalities in children. ${ }^{35}$ Our study further suggests that public health interventions to prevent SES gradients in CVD risk should focus on reducing childhood obesity and promote physical fitness in all children, with special focus on girls and low-SES groups.

\section{References}

1. Wielgosz A, Arango M, Bancej C, et al. editors. 2009 Tracking heart disease and stroke in Canada [Internet]. Ottawa (ON): Public Health Agency of Canada; 2009 [cited 2015 Feb 26]. Available from: http:// www.phac-aspc.gc.ca/publicat/2009/cvd-avc/ pdf/cvd-avs-2009-eng.pdf

2. Choiniere R, Lafontaine $P$, Edwards AC. Distribution of cardiovascular disease risk factors by socioeconomic status among Canadian adults. CMAJ. 2000;162 (9 Suppl): S13-S24.

3. Millar WJ, Wigle DT. Socioeconomic disparities in risk factors for cardiovascular disease. CMAJ. 1986;134(2):127-32. 
4. Batty GD, Leon DA. Socio-economic position and coronary heart disease risk factors in children and young people. Evidence from UK epidemiological studies. Eur J Public Health.2002;12(4):263-72.

5. Boreham C, Twisk J, van Mechalen W, Savage M, Strain J, Cran G. Relationships between the development of biological risk factors for coronary heart disease and lifestyle parameters during adolescence: The Northern Ireland Young Hearts Project. Public Health. 1999;113(1):7-12.

6. Elgar FJ, Pfortner TK, Moor I, De CB, Stevens $\mathrm{GW}$, Currie C. Socioeconomic inequalities in adolescent health 2002-2010: a time-series analysis of 34 countries participating in the Health Behaviour in School-aged Children study. Lancet. 2015;385(9982):2088-95.

7. Janssen I, Boyce WF, Simpson K, Pickett W. Influence of individual- and area-level measures of socioeconomic status on obesity, unhealthy eating, and physical inactivity in Canadian adolescents. Am J Clin Nutr. 2006;83(1):139-45

8. Simen-Kapeu A, Veugelers PJ. Socio-economic gradients in health behaviours and overweight among children in distinct economic settings. Can J Public Health. 2010;101(Suppl 3):S32-6.

9. Shonkoff JP, Boyce WT, McEwen BS. Neuroscience, molecular biology, and the childhood roots of health disparities: building a new framework for health promotion and disease prevention. JAMA. 2009;301(21): 2252-9. DOI: 10.1001/jama.2009.754.

10. Non AL, Rewak M, Kawachi I, et al. Childhood social disadvantage, cardiometabolic risk, and chronic disease in adulthood. Am J Epidemiol. 2014;180(3):263-71. DOI: 10.1093/ aje/kwu.127.

11. Wannamethee SG, Whincup PH, Shaper G, Walker M. Influence of fathers' social class on cardiovascular disease in middle-aged men. Lancet. 1996;348(9037):1259-63.

12. Chen E, Martin AD, Matthews KA. Trajectories of socioeconomic status across children's lifetime predict health. Pediatrics. 2007;120(2):e297-303.

13. Bradley RH, Corwyn RF. Socioeconomic status and child development. Annu Rev Psychol. 2002(53):371-99.
14. Richter M, Moor I, van Lenthe FJ. Explaining socioeconomic differences in adolescent self-rated health: the contribution of material, psychosocial and behavioural factors. J Epidemiol Community Health. 2012;66 (8):691-7. DOI: $10.1136 /$ jech.2010.125500.

15. Bambra CL, Hillier FC, Moore HJ, Summerbell $\mathrm{CD}$. Tackling inequalities in obesity: a protocol for a systematic review of the effectiveness of public health interventions at reducing socioeconomic inequalities in obesity amongst children. Syst Rev. 2012;1:16 DOI: 10.1186/ 2046-4053-1-16.

16. Veugelers PJ, Fitzgerald AL. Prevalence of and risk factors for childhood overweight and obesity. CMAJ. 2005;173(6):607-13.

17. Hajizadeh M, Campbell MK, Sarma S. Socioeconomic inequalities in adult obesity risk in Canada: trends and decomposition analyses. Eur J Health Econ. 2014;15(2): 203-21. DOI: $10.1007 /$ s10198-013-0469-0.

18. Howe LD, Lawlor DA, Propper C. Trajectories of socioeconomic inequalities in health, behaviours and academic achievement across childhood and adolescence. J Epidemiol Community Health. 2013;67(4):358-64.

19. McCrindle BW, Manlhiot C, Millar K, et al. Population trends toward increasing cardiovascular risk factors in Canadian adolescents. J Pediatr. 2010;157(5):837-43.

20. Van Lenthe FJ, Boreham CA, Twisk JW, Strain JJ, Savage JM, Smith GD. Socioeconomic position and coronary heart disease risk factors in youth. Findings from the Young Hearts Project in Northern Ireland. Eur J Public Health. 2001;11(1):43-50.

21. Tremblay M, Wolfson M, Connor GS Canadian Health Measures Survey: rationale, background and overview. Health Rep. 2007;18(Supp 1): 7-20.

22. Canadian Health Measures Survey (CHMS): data user guide: Cycle 2 [Internet]. Ottawa (ON): Statistics Canada; 2012 Nov [cited 2015 Feb 26]. Available from http://data library utoronto ca/datapub/codebooks/cstdli/chms/ CHMS_User_Guide_Cycle2_E pdf2012

23. Craig CL, Marshall AL, Sjostrom M, et al. International physical activity questionnaire: 12-country reliability and validity. Med Sci Sports Exerc. 2003;35(8):1381-95
24. de Onis M, Onyango AW, Borghi E, Siyam A, Nishida C, Siekmann J. Development of a WHO growth reference for school-aged children and adolescents. Bull World Health Organ. 2007;85(9):660-7.

25. Canadian Society for Exercise Physiology (CSEP). The Canadian Physical Activity, Fitness and Lifestyle Approach (CPAFLA): CSEP-Health \& Fitness Program's HealthRelated Appraisal and Counselling Strategy, 3rd ed. Ottawa (ON): Canadian Society for Exercise Physiology; 2003.

26. Shields M, Tremblay MS, Laviolette M, Craig CL, Janssen I, Connor Gorber S. Fitness of Canadian adults: results from the 2007-2009 Canadian Health Measures Survey. Health Rep. 2010;21(1):21-35.

27. Tremblay MS, Shields M, Laviolette M, Craig CL, Janssen I, Connor Gorber S. Fitness of Canadian children and youth: results from the 2007-2009 Canadian Health Measures Survey. Health Rep. 2010;21(1):7-20.

28. Shi Y, de Groh M, Morrison H. Increasing blood pressure and its associated factors in Canadian children and adolescents from the Canadian Health Measures Survey. BMC Public Health. 2012;12(1):388. DOI: 10.1186/ 1471-2458-12-388.

29. Lee JM, Okumura MJ, Davis MM, Herman WH, Gurney JG. Prevalence and determinants of insulin resistance among U.S. adolescents: a population-based study. Diabetes Care. 2006;29(11):2427-32.

30. Sobal J, Stunkard AJ. Socioeconomic status and obesity: a review of the literature. Psychol Bull. 1989;105(2):260-75.

31. Shields M. Overweight and obesity among children and youth. Health Rep. 2006;17(3): 27-42.

32. Hajizadeh M, Campbell MK, Sarma S. Socioeconomic inequalities in adult obesity risk in Canada: trends and decomposition analyses. Eur J Health Econ. 2014;15(2):20321. DOI: $10.1007 / \mathrm{s} 10198-013-0469-0$.

33. Matheson FI, Moineddin R, Glazier RH. The weight of place: a multilevel analysis of gender, neighborhood material deprivation, and body mass index among Canadian adults. Soc Sci Med. 2008;66(3):675-90. 
34. Bohr AD, Brown DD, Laurson KR, Smith PJ, Bass RW. Relationship between socioeconomic status and physical fitness in junior high school students. J Sch Health. 2013;83 (8):542-7. DOI: 10.1111/josh.12063.

35. Creating a healthier Canada: making prevention a priority: a declaration on prevention and promotion from Canada's Ministers of Health and Health Promotion/ Healthy Living [Internet]. Ottawa (ON): Public Health Agency of Canada; [modified 2010 Sep 7; cited 2015 Mar 4]. Available from: http://www.phac-aspc.gc.ca/hp-ps/ hl-mvs/declaration/index-eng.php

36. Bergstrom E, Hernell O, Persson LA. Cardiovascular risk indicators cluster in girls from families of low socio-economic status. Acta Paediatr. 1996;85(9):1083-90.

37. Jimenez-Pavon D, Kelly J, Reilly JJ. Associations between objectively measured habitual physical activity and adiposity in children and adolescents: systematic review. Int J Pediatr Obes. 2010;5(1):3-18. DOI: 10.3109/ 17477160903067601 .

38. Ness AR, Leary SD, Mattocks C, et al. Objectively measured physical activity and fat mass in a large cohort of children. PLoS Med. 2007;4(3):e97.

39. Carson V, Spence JC, Cutumisu N, Cargill L. Association between neighborhood socioeconomic status and screen time among pre-school children: a cross-sectional study. BMC Public Health. 2010;10:367 DOI: 10.1186/1471-2458-10-367.

40. Fairclough SJ, Boddy LM, Hackett AF, Stratton G. Associations between children's socioeconomic status, weight status, and sex, with screen-based sedentary behaviours and sport participation. Int J Pediatr Obes. 2009;4(4): 299-305. DOI: 10.3109/17477160902811215.

41. Denton SJ, Trenell MI, Plotz T, Savory LA, Bailey DP, Kerr CJ. Cardiorespiratory fitness is associated with hard and light intensity physical activity but not time spent sedentary in 10-14 year old schoolchildren: the HAPPY study. PLoS One. 2013;8(4):e61073. DOI: 10.1371/journal.pone.0061073.

42. Phillips JA, Young DR. Past-year sports participation, current physical activity, and fitness in urban adolescent girls. J Phys Act Health. 2009;6(1):105-11.
43. Lehman BJ, Taylor SE, Kiefe CI, Seeman TE. Relationship of early life stress and psychological functioning to blood pressure in the CARDIA study. Health Psychol. 2009; 28(3):338-46. DOI: 10.1037/a0013785.

44. Pulkki L, Keltikangas-Jarvinen L, Ravaja N, Viikari J. Child-rearing attitudes and cardiovascular risk among children: moderating influence of parental socioeconomic status. Prev Med. 2003;36(1):55-63.

45. Donin AS, Nightingale CM, Owen CG, et al. Ethnic differences in blood lipids and dietary intake between UK children of black African, black Caribbean, South Asian, and white European origin: the Child Heart and Health Study in England (CHASE). Am J Clin Nutr. 2010;92(4):776-83. DOI: 10.3945/ ajcn.2010.29533.

46. Freedman DS, Strogatz DS, Williamson DF, Aubert RE. Education, race, and high-density lipoprotein cholesterol among US adults. Am J Public Health. 1992;82(7):999-1006.

47. Juhola J, Magnussen CG, Viikari JS, et al. Tracking of serum lipid levels, blood pressure, and body mass index from childhood to adulthood: the Cardiovascular Risk in Young Finns Study. J Pediatr. 2011;159(4): 584-90. 